Rev. salud pública. 10 sup (1): 58-71, 2008

\title{
Equidad de Etnia en el Acceso a los Servicios de Salud en Bogotá, Colombia, 2007
}

\author{
Ethnic equity in accessing health services in Bogotá, Colombia, 2007 \\ John F. Ariza-Montoya y Mario E. Hernández-Álvarez \\ Universidad Nacional de Colombia. Sede Bogotá, jfarizam@unal.edu.co, mehernandeza@unal.edu.co \\ Recibido 2 Marzo 2008/Enviado para Modificación 23 Abril/Aceptado 12 Mayo 2008
}

\section{RESUMEN}

Objetivo Identificar inequidades en el acceso a los servicios de salud derivadas de la pertenencia étnica en Bogotá.

Metodología En el 2007 se realizaron 39 entrevistas a profundidad y seis grupos focales con integrantes de los grupos étnicos. Los hallazgos cualitativos se contrastaron con los resultados del procesamiento estadístico de datos provenientes de la Encuesta Calidad de Vida 2003 y la ficha de caracterización de la estrategia de Atención Primaria en Salud, denominada Salud a su Hogar en Bogotá, discriminando variables demográficas, socioeconómicas, de necesidades, acceso y resultados en salud.

Resultados Se caracterizaron las siguientes etnias: indígenas, negros, raizales isleños y gitanos. Se documentaron diferencias a nivel socioeconómico, educativo, laboral, en el acceso al aseguramiento, utilización de servicios y resultados en salud, que se consideran inequidades relacionadas con las siguientes condiciones determinantes: 1.Discriminación étnica y racial. 2. Diferencias en el estatus social, económico y político y violación de derechos. 3. Interacciones entre inmigración, aculturación y asimilación.

Conclusiones Existen inequidades derivadas de la pertenencia étnica en Bogotá en el acceso a los servicios de salud expresadas en desigualdades en el acceso según las necesidades, indicios de violación de derechos, discriminación negativa y falta de adecuación y reconocimiento de las diferencias étnicas.

Palabras Clave: Equidad en salud, grupos étnicos, acceso a servicios, evaluación de servicios de salud, equidad en el acceso (fuente: DeCS, BIREME).

\section{ABSTRACT}

Objective Identifying inequalities in gaining access to health services resulting from ethnicity in Bogota.

Methods 39 in-depth interviews with focus groups and six members of ethnic groups were conducted during 2007. Qualitative findings were contrasted with the results 
from statistically processing data from the Quality of Life Survey 2003 and characterising the Primary Health Care strategy called "Health in your Home in Bogota", discriminating the following variables: demographic, socio-economic, needs, access and health outcomes.

Results The following ethnic groups were characterised: indigenous people, black people, gypsies and islanders. Differences in socioeconomic status, education, employment, access to health insurance, use of health services and outcomes were documented as these were considered to be inequities related to the following determinants: ethnic and racial discrimination, differences in social, economic and political status and violation of rights, interactions between immigration, acculturation and assimilation and differentials exposure.

Conclusions There are ethnic inequities in gaining access to health services because there is no adequate access as required; there is violation of rights, discrimination, a lack of adaptation and appreciation of differences. These situations are considered to be examples of cultural and distributive injustice. Ethnicity determines levels of social vulnerability and takes specific forms regarding life, health and disease, thereby becoming a structural determinant of studying ethnic-equity in gaining access to health services.

Key Words: Equity in health, ethnic group, access to and evaluation of health services, equity of access (source: MeSH, NLM).

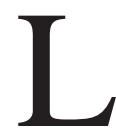

a pertenencia a un grupo étnico se ha considerado como uno de los principales determinantes en la configuración de desigualdades indeseables e injustas en salud (1,2). Según datos del Censo 2005 del DepartamentoAdministrativo Nacional de Estadística, en Colombia las etnias representan el 13,8 \% de la población, mientras que en Bogotá representan el 1,7 \%, con la siguiente distribución: 15032 indígenas, 523 Gitanos o del Pueblo Rom y 98002 afro descendientes, que incluyen a 96490 negros continentales, 157 palenqueros de Palenque de San Basilioy 1355 raizales isleños. Se define etnicidad como la pertenencia a un grupo étnico, entendido como un colectivo que ha mantenido y actualizado su identidad, refiere un origen y unas características que pueden estar o no presentes, como el idioma, gobierno propio, rasgos físicos y niveles comunes de acceso a servicios (3). Aunque la raza, como categoría colonialista se ha venido asociando a diferencias fundamentadas en factores biológicos, los últimos estudios genéticos no apoyan esta segregación y plantean que no hay razas puras (4). En Colombia, el concepto de raza ha sido acogido por el movimiento negro, para visibilizar la segregación histórica (5). En el presente estudio, la raza y la etnia se consideran productos históricos y culturales, sobre los cuales ha recaído una fuerte carga de violencia y discriminación. Solo hasta 2005 se incorporaron las variables de las cuatro etnias en un censo nacional en Colombia, y actualmente no se cuenta con información diferenciada en el sector salud. Bogotá es un receptor importante de grupos étnicos como conse- 
cuencia del desplazamiento forzado y el conflicto armado, así como por desastres naturales, falta de oportunidades de estudio y trabajo, dificultades económicas y bajo nivel de servicios en el lugar de origen.

En la región de las Américas se han documentado diferencias socioeconómicas de los grupos étnicos respecto de la población general $(6,7)$, menor acceso a servicios, menor ingreso, mayores niveles de morbilidad y mortalidad (8). En este estudio se entiende que tales diferencias dependen de la construcción de la etnicidad y de la posición social derivada de ella. Varios estudios de equidad en salud han incorporado las variables de etnia y raza $(2,4,9)$ y han demostrado que los grupos étnicos mantienen de manera sistemática diferencias en el acceso a los servicios de salud, aún después de controlar otras variables (10). Las explicaciones están relacionadas con los patrones sociales, culturales y de discriminación, y no tanto con la susceptibilidad genética. La discriminación se entiende como la distribución desigual del poder social, económico y político, que determina que grupos poblacionales específicos estén explotados y marginados de la posibilidad de construir un desarrollo propio y un nivel satisfactorio de autonomía y ejercicio de sus derechos.

La inequidad en salud se entiende en este estudio (11) como la presencia de desigualdades en salud, enfermedad y muerte, derivada de una desventaja sistemática de un grupo social respecto de otros como resultado de su posición social. Tal posición resulta de las diferencias de poder, riqueza o prestigio, definidas por su lugar en la estructura laboral o socioeconómica, de género, de raza, de etnia o del ciclo vital en que se encuentra. Un referente inmediato para valorar una desigualdad como inequidad es la falta sistemática de garantía de derechos humanos interdependientes, en especial a la salud, definidos en el pacto constitucional y en los pactos internacionales de derechos humanos. También existe una inequidad en el acceso a los servicios de salud cuando éstos no reconocen las diferencias o no responden a diferenciales de necesidad. Se adopta la postura de Fraser (12), en relación a que la equidad debe incorporar políticas de redistribución frente a desigualdades injustas, tanto como de reconocimiento, frente a las diferencias derivadas de la identidad. La exclusión derivada de la etnia se asocia con frecuencia a otras exclusiones. De allí que la equidad de etnia en salud está dirigida a que los grupos étnicos tengan las mismas oportunidades que el resto de la población para acceder y utilizar los servicios. Pero también, los servicios de salud deben reconocer las necesidades y potencialidades diferentes de los grupos étnicos, derivadas de sus propios conceptos de salud (13). Se entiende en este trabajo el acceso a servicios de salud como el "proceso mediante el cual puede lograrse que una necesidad de atención, bien sea por el deterioro en el 
estado de salud, un diagnóstico sobre el mismo o la promoción de su mejoramiento, se satisfaga completamente" (10). Al respecto, se reconoce la importancia del aseguramiento en salud, como uno de los determinantes del acceso a los servicios de salud, pero no el único. Al mismo tiempo, es clara la importancia de estudiar las diferencias culturales, considerando el acceso como un proceso más allá del contacto inicial con el sistema de salud y verificar los demás aspectos que intervienen en el uso real y efectivo de servicios $(14,15)$.

\section{METODOLOGÍA}

Siguiendo a Sen (16), en este estudio se intenta contrastar la percepción de la población con datos registrados en otras fuentes de información. Este ejercicio tiene mayor relevancia en la documentación de inequidades en los grupos étnicos, quienes plantean la existencia de prácticas de discriminación por parte de los servicios de salud que sólo son sentidas por quien las padece. El enfoque cualitativo permite entender cómo operan los factores que afectan el acceso y se desarrolló en este estudio a través de entrevistas a profundidad y grupos focales. Para el análisis se definieron las siguientes categorías: identidades étnicas; necesidades y potencialidades en salud; acceso a servicios; sugerencias de la población. Durante el año 2007, se realizaron 39 entrevistas a profundidad y seis grupos focales con integrantes de grupos étnicos y funcionarios. La información se sistematizó con la ayuda del software de análisis cualitativo NVIVO versión 7, que permite interpretar textos y contribuir a la formulación de teorías a través de la extracción del conocimiento conceptual de los documentos. El proceso de categorización de entrevistas y grupos focales implicó varias fases: separación de códigos; identificación y clasificación de códigos por familias; síntesis y agrupamiento. Se validaron los datos con los entrevistados y se realizó contrastación de las diferentes fuentes (17).

Como parte de la visión cuantitativa se realizó el análisis de la Encuesta de Calidad de Vida (ECV) del año 2003 y de una sección de la sistematización de la ficha de caracterización de la población incluida en la estrategia de Atención Primaria en Salud (APS) con enfoque familiar y comunitario de la Secretaría Distrital de Salud, denominada «Salud a su Hogar» (SASH). Esta población habita en los estratos socioeconómicos 1 y 2 de la ciudad y fue caracterizada en diferentes momentos entre el tercer trimestre de $2004 \mathrm{y}$ el primer trimestre de 2007. Dentro de las opciones de medición de desigualdades en el acceso a los servicios de salud se realizó la comparación directa entre grupos diferenciados por la pertenencia étnica, incorporando covariables de comparación, como sexo, edad, ingreso, educación, empleo, tenencia de vivienda, calidad del 
agua, disponibilidad de alimentos y riesgos ambientales cercanos a la vivienda, con el fin de visibilizar la interacción entre estas variables y la importancia de la pertenencia étnica en la generación de las desigualdades. Esta investigación incluye dentro de su definición de accesibilidad, los procesos de afiliación al Sistema General de Seguridad Social en Salud (SGSSS) como componente del nivel de acceso potencial y el financiamiento de los servicios.

Se realizó un análisis descriptivo estratificado por etnia con pruebas de significancia (Chi Cuadrado y Análisis de Varianza). El procesamiento se llevó a cabo por medio del paquete estadístico SPSS versión 10.

\section{RESULTADOS}

La proporción de las etnias en la población cambia de acuerdo a la fuente utilizada (Tabla 1). El auto-reconocimiento como principal herramienta para el registro estadístico, expresa el nivel de conciencia étnica. Los entrevistados asumen la etnicidad como una categoría de pertenencia a un grupo que se diferencia por tener usos y costumbres diferentes, y que puede o no incluir rasgos como el idioma, el vestido, mecanismos específicos de regulación social, rituales, entre otros. En el caso de la población gitana la etnicidad constituye un entorno poco permeable y los raizales isleños se diferencian de los negros continentales por su historia y su cultura.

“...ser gitano es de nacimiento...” Entrevista hombre gitano de 54 años.

“...no se es negro por el color de piel... es por la militancia, por la defensa activa de los derechos..." Entrevista mujer negra de 41 años.

"...somos de pueblos con mucha memoria... haber compartido hasta la misma teta, es compartir la historia, la memoria..." Entrevista hombre negro de 21 años.

Tabla 1. Distribución de población según pertenencia étnica en Bogotá

\begin{tabular}{lcccccc}
\hline \multirow{2}{*}{ Etria } & $\begin{array}{c}\text { Censo poblacional } \\
\text { 2005 }\end{array}$ & \multicolumn{2}{c}{$\begin{array}{c}\text { Muestra } \\
\text { ponderada } \\
\text { de ECV 2003 }\end{array}$} & \multicolumn{2}{c}{$\begin{array}{c}\text { Muestra real } \\
\text { de SASH }\end{array}$} \\
\cline { 2 - 7 } & Casos & $\%$ & Casos & $\%$ & Casos & $\%$ \\
\hline Indigena & 15032 & 0,22 & 3623 & 0,05 & 2004 & 0,18 \\
Rom o gitano & 523 & 0,01 & 2956 & 0,04 & 2274 & 0,21 \\
Raizal isleño & 1355 & 0,02 & 720 & 0,01 & - & - \\
Negro (a) & 96530 & 1,42 & 75410 & 1,1 & 3330 & 0,31 \\
Ninguno de los anteriores & 6450329 & 95,2 & 6744646 & 98,8 & 1085035 & 99,3 \\
No Informa & 214922 & 3,2 & - & - & - & - \\
\hline Total & 6778691 & 100 & 6819637 & 100 & 1092743 & 100 \\
\hline
\end{tabular}

Fuente: DANE y ECV 2003. Cálculos propios 
Uno de los escenarios de reconocimiento de las etnias en la ciudad son los territorios simbólicos. Los indígenas están conformados en su mayoría por grupos ancestrales Muiscas, pero otros grupos han inmigrado a la ciudad desde varias regiones del país.

“ ...con el origen, con la historia y la palabra...” Grupo focal población indígena.

“ ...los negros comparten un territorio simbólico... hay sitios en donde están los negros, donde se unen, donde se encuentran...” Entrevista hombre negro de 53 años.

Tabla 2. Comparación de algunos indicadores socioeconómicos (\%) entre los grupos étnicos y la población sin pertenencia étnica en Bogotá. ECV-2003

\begin{tabular}{|c|c|c|c|c|c|c|}
\hline \multirow[b]{2}{*}{$\begin{array}{c}\text { Indicador } \\
\text { (Proporción \%) }\end{array}$} & \multicolumn{5}{|c|}{ Población } & \multirow[b]{2}{*}{$\begin{array}{l}\text { Valor } \\
\text { de P }\end{array}$} \\
\hline & Negra & Indigena & Gitana & Raizal & $\begin{array}{c}\text { Sin } \\
\text { pertenencia } \\
\text { étnica }\end{array}$ & \\
\hline $\begin{array}{l}\text { Población que se percibe } \\
\text { como pobre. }\end{array}$ & 54,7 & 53,5 & 73,0 & 68,5 & 48,0 & 0 \\
\hline $\begin{array}{l}\text { Población pobre según } \\
\text { linea de pobreza }\end{array}$ & 36,9 & 40,7 & 35,3 & 54,7 & 35 & 0.001 \\
\hline $\begin{array}{l}\text { Población en niveles } 1 \text { y } \\
2 \text { del SISBEN }\end{array}$ & 45,5 & 41,1 & 54,4 & 28,8 & 39,3 & 0,001 \\
\hline $\begin{array}{l}\text { Población sin ningún } \\
\text { nivel educativo }\end{array}$ & 2,9 & 6,2 & 5,2 & 0 & 2,3 & $<0,00$ \\
\hline $\begin{array}{l}\text { Población con } \\
\text { analfabetismo }\end{array}$ & 3,2 & 5,5 & 5,2 & 0 & 3,9 & $<0,00$ \\
\hline $\begin{array}{l}\text { Población con nivel } \\
\text { universitario }\end{array}$ & 9,0 & 9,9 & 10,8 & 8,5 & 14,1 & $<0,00$ \\
\hline $\begin{array}{l}\text { Población que está } \\
\text { buscando trabajo }\end{array}$ & 5,3 & 5,5 & 2,1 & 0 & 5,1 & $<0,00$ \\
\hline $\begin{array}{l}\text { Población que reporta } \\
\text { ser jornalero o peón. }\end{array}$ & 2,4 & 0 & 0 & 0 & 0,1 & $<0,00$ \\
\hline $\begin{array}{l}\text { Población que reporta } \\
\text { ser patrón. }\end{array}$ & 2,3 & 1,8 & 0 & 0 & 3,8 & $<0,00$ \\
\hline $\begin{array}{l}\text { Población que no tiene } \\
\text { contrato de trabajo } \\
\text { (informalidad). }\end{array}$ & 35,5 & 27,7 & 94,7 & 47,4 & 34,7 & $<0,00$ \\
\hline $\begin{array}{l}\text { Hogares con riesgos } \\
\text { cercanos a la vivienda }\end{array}$ & 30,5 & 38,7 & 41,2 & 24,9 & 33,1 & $<0,00$ \\
\hline $\begin{array}{l}\text { Hogares con mala } \\
\text { calidad del agua }\end{array}$ & 9,2 & 16,7 & 3,7 & 5,4 & 14,7 & $<0,00$ \\
\hline $\begin{array}{l}\text { Hogares que ha dejado } \\
\text { de consumir las tres } \\
\text { comidas al dia por falta } \\
\text { de dinero. }\end{array}$ & 5,5 & 9,7 & 14,3 & 0 & 8,7 & $<0,00$ \\
\hline
\end{tabular}

En la población sin pertenencia étnica los hombres representan el 53,2 \%, mientras que en las etnias el grupo mayoritario son las mujeres, con una frecuencia de 50,5 \% en negros, 53,6 \% en indígenas, $61 \%$ en gitanos y 66,1 $\%$ en raizales. Las etnias tienen menor proporción de población en los grupos más jóvenes y mayor en el grupo de 45 a 60 años, que la población general. Algunas condiciones socioeconómicas percibidas y objetivas de los 
grupos aparecen en la Tabla 2. Estos grupos perciben y viven una concentración de factores de vulnerabilidad que configura una posición social de desventaja.

“...En la ciudad todo cambió...porque es que no tenemos un salario, no es de nuestra cultura, un ingreso fijo..." Entrevista hombre gitano de 37 años.

“...uno no puede llegar muy tarde, es peligroso... no podemos mandar a los niños a estudiar porque les queda muy lejos... a la mujer la manosean... ya esa muchacha está con eso del vicio..." Grupo focal población indígena.

“...aguantamos hambre... teníamos comida allá en la tierra... acá no se puede trabajar, apenas algo de artesanías..." Grupo focal con población indígena.

El desplazamiento por el conflicto armado es una de las causas de las precarias condiciones. Según el gobierno nacional, en Bogotá se encuentran 7.724 negros y 5.474 indígenas en situación de desplazamiento que corresponden al 3,9 \% y 2,8 \% respectivamente de este grupo en el distrito. Esta cifra contrasta con los hallazgos de una investigación realizada por la Secretaria Distrital de Salud y la Universidad Javeriana que reportó un 6,3 \% de negros y 4,5 \% de indígenas del total de desplazados en Bogotá.

“... ahora ya no se puede estar en nuestra casa, en la maloca... es mucha violencia, nos han matado a nuestros líderes...” Entrevista hombre Kankuamo de 42 años.

Se evidencia la permanencia del racismo, expresado en prejuicios cotidianos y marginación de instancias de poder. Los gitanos se han estigmatizado como ladrones, embusteros, ladrones, portadores de enfermedades y brujería. Negros e indígenas se asocian injustamente con mínimas capacidades mentales, con el bullicio y la falta de higiene.

“...a nosotras nos tratan muy mal, cuando nos ven en la calle a veces nos gritan cosas feas, así que brujas, mentirosas....” Grupo focal con población gitana.

“... el tipo tuvo que renunciar... por lo menos dos veces por semana se lo llevaban al calabozo solo por ser negro....” Grupo focal con población negra.

Los grupos conciben los servicios de salud como una opción secundaria para la atención de enfermedades. Para la población negra la salud está relacionada a las capacidades que permiten estar alegre, participar en las actividades colectivas. La enfermedad se oculta porque se considera que no se debe socializar con extraños ni darle importancia. 
“... la etnia vive feliz, porque vive sonriendo y por esa alegría la gente no se enferma..." Grupo focal con población negra.

La concepción del equilibrio natural que implica la construcción de relaciones con los seres espirituales expresa la concepción de salud para la población indígena y determinan múltiples restricciones y prohibiciones. La salud está relacionada con la alimentación y con los buenos hábitos en la dieta.

“...lo más importante es una relación espiritual con la madre tierra...” Grupo focal con población Muisca.

“...Ahora no podemos comer pescado de cuero y tampoco carne de res ni marrano, porque necesito completar mi dieta... cuando tiene menstruación no puede salir mucho ni ver enfermo ni nada, y tampoco puede bañar en el río...” Grupo focal con indígenas Tugus y Huitotos. “... Acá uno se enferma fácil, por cualquier cosa, que la comida, que el viento, todo lo enferma a uno....” Entrevista hombre Huitoto 38 años

La población raizal que vive en Bogotá relaciona la salud con la posibilidad de acceder a los servicios, pues la mayoría viene a estudiar, y con la capacidad de realizar sus actividades culturales y religiosas.

“...nosotros no tenemos la medicina tradicional... la salud es estar sano, sin enfermedades, y nosotros casi todos como que tenemos el seguro, aunque no se ha realizado un estudio específico... la salud también es estar alegre, tranquilos, unidos y poder estar en paz espiritual en la iglesia...” Entrevista mujer raizal de 38 años.

La población gitana refiere un bajo nivel de auto percepción de necesidades en salud, lo cual se explica por mecanismos tradicionales de prevención.

“...Nosotros tenemos el marimé como un conjunto de elementos para mantener sanos, como de prevención, para no mezclarnos... es como un aseo, una higiene con todas las cosas...” Entrevista mujer gitana de 34 años.

La población indígena y gitana reporta con mayor frecuencia la presencia de enfermedad crónica, necesidad sentida en el último mes y un mal estado de salud, con respecto a la población sin pertenencia étnica. De igual manera, los cuatro grupos étnicos refieren con mayor frecuencia tener diagnóstico de bajo peso, hipertensión arterial y presencia de alguna limitación (Tabla 3). La importancia asignada por los grupos étnicos a los servicios de salud y al aseguramiento varía en función de la presencia de necesidades en salud, de la condición socio-económica, de la fortaleza de la cultura tradicional y de las experiencias anteriores de la población con los servicios de salud. Se 
tiene la percepción de que los servicios de salud asumen una función restrictiva e impositiva.

“...cuando los sabedores no pueden, se complementan... confían más en las enseñanzas y las prácticas médicas tradicionales que en el médico occidental..." Entrevista hombre y líder Muisca de 36 años. “... a la gente no le gusta ir donde el médico...” Entrevista hombre gitano de 37 años.

Tabla 3. Comparación de indicadores de necesidades sentida en salud entre los grupos étnicos y la población sin pertenencia étnica

\begin{tabular}{|c|c|c|c|c|c|c|}
\hline \multirow[b]{2}{*}{$\begin{array}{c}\text { Indicador } \\
\text { (Proporción \$s) }\end{array}$} & \multicolumn{5}{|c|}{ Poblacion } & \multirow[b]{2}{*}{$\begin{array}{c}\text { Valer de } \\
\mathrm{P}\end{array}$} \\
\hline & Negra & Indigena & Gitana & Raizal & $\begin{array}{c}\text { Sin } \\
\text { pertenencia } \\
\text { Atrica }\end{array}$ & \\
\hline $\begin{array}{l}\text { Poblacién que reporta } 8 u \\
\text { estado de salud como malo }\end{array}$ & 15,3 & 34,1 & 40,6 & 35,1 & 21,0 & 0 \\
\hline $\begin{array}{l}\text { Población con necesidad } \\
\text { gentida en selud en el ùltime } \\
\text { mes }\end{array}$ & 9,3 & 11,6 & 33,4 & 2,1 & 9,2 & $<0,000$ \\
\hline $\begin{array}{l}\text { Población que refiere tener } \\
\text { diegnobsfico de enfermedad } \\
\text { crónica }\end{array}$ & 10,1 & 26,3 & 41,0 & 10,3 & 14,0 & $<0,000$ \\
\hline $\begin{array}{l}\text { Población que refiere tener una } \\
\text { enfermedad grane }\end{array}$ & 5,6 & 122 & 5,4 & 7,5 & 10.7 & 0.001 \\
\hline $\begin{array}{l}\text { Poblaciên can necesidad } \\
\text { sentida en salud (SASH) }\end{array}$ & 22,3 & 21,5 & 10,6 & ND & 17,3 & $\varangle 0,000^{\circ}$ \\
\hline $\begin{array}{l}\text { Población que refiere tener } \\
\text { diegnobstico de bajo peso } \\
\text { (S/SH) }\end{array}$ & 3,1 & 3,5 & 3,5 & ND & 1,9 & $0,009^{\circ}$ \\
\hline $\begin{array}{l}\text { Poblacion que refiere tener } \\
\text { diegnobsico de diabetes } \\
(\mathrm{SASH})\end{array}$ & 1,8 & 3,2 & 1,5 & ND & 1,5 & $40,000^{\circ}$ \\
\hline $\begin{array}{l}\text { Poblacion que refiere tener } \\
\text { disgnobstico de hipertensión } \\
\text { arterial (SASH) }\end{array}$ & 5,8 & 5,9 & 5.6 & ND & 5,1 & $<0,000^{\circ}$ \\
\hline $\begin{array}{l}\text { Poblacion que refiere tener } \\
\text { limitación [SASH] }\end{array}$ & 2,3 & 3.2 & 1,8 & ND & 1,4 & $<0,000^{\circ}$ \\
\hline
\end{tabular}

A diferencia de negros y raizales, los indígenas y gitanos sí son considerados como poblaciones especiales en el Sistema General de Seguridad Social en Salud (SGSSS), lo cual les otorga algunos beneficios, tales como la prioridad en la afiliación al Régimen Subsidiado y la exención en el pago de copagos y cuotas de recuperación para indígenas y gitanos. El SGSSS ha incorporado un "régimen especial” para la población indígena, con acciones específicas como la adecuación sociocultural de los servicios, el ajuste de los planes de beneficios para incluir las medicinas tradicionales, el pago de sobrecostos hasta del $50 \%$ en el valor de la prima para el asegurador por cada afiliado indígena, la posibilidad de que las organizaciones indígenas conformen sus propias Empresas Promotoras de Salud (EPS) e Instituciones Prestadoras de Salud (IPS), 
entre otros. A pesar de estas acciones afirmativas, la población indígena entrevistada considera que el aseguramiento es una burla y una imposición que no ha traído buenos resultados.

“...como somos indígenas les dan más plata y por eso corren a meternos en una lista, pero eso es puro negocio, después se pierden y cumplen nada y atienden mal y uno coloca quejas y no pasa nada, nos cogen es de bobos..." Entrevista hombre Muisca de 32 años.

La población negra es la etnia con menor cobertura del seguro de salud, aunque percibe más necesidades en salud que las demás poblaciones (Tabla 4). Las poblaciones indígenas y gitanas tienen mayor cobertura en la afiliación al Sistema General de Seguridad Social en Salud (SGSSS), pero refieren barreras para acceder a los servicios en caso de necesitarlos y se presenta discriminación en los servicios con acciones de burla, violencia, negación y aplazamiento de la atención. Los procesos administrativos presentan trámites excesivos que producen rechazo. En la consulta médica la población siente que se le presume culpable de su enfermedad, que no existen procesos de adecuación de los servicios y que se rechazan sus concepciones de salud y enfermedad. La discriminación se expresa en el bajo número de personas de los grupos que son contratadas por los aseguradores y prestadores en la ausencia de sistemas específicos de información. La ausencia de dinero es otra barrera frecuente.

“...la apariencia genera rechazo y burla...” Entrevista mujer gitana de 34 años

“... a uno le hablan con agresividad, le hablan duro y como con asco, a veces se aguanta y a veces no..." Entrevista hombre negro de 33 años.

“...a uno lo discriminan cuando los médicos se burlan de uno, por sus apellidos, porque uno le dice que es indígena...” Entrevista mujer Muisca de 37 años.

“...suelen decirle a uno es que a ustedes los negros les da esta enfermedad... no se cuidan... es que ustedes todos tienen anemia..." Entrevista mujer negra de 41 años.

“...uno a veces no tiene esa plata, para hacerse los exámenes, medicamentos... no hay con quien dejar a los niños... el transporte...” Grupo focal con población negra.

Existen cuatro grupos con diferentes posibilidades económicas para acceder a los servicios: 1 . Cabildos indígenas y Kumpania gitana registrada. 2. Desplazados que están registrados en el registro de la Agencia Presidencial para la Acción Social y la Cooperación Internacional “Acción Social”. 3. Desplazados que no están registrados. 4. Otros (Integrantes de grupos étnicos no reconocidos por el Estado, migrantes, estudiantes). Sólo los dos primeros grupos son catalogados como población especial dentro del SGSSS, lo que 
implica afiliación al Régimen Subsidiado y no tener que pagar copagos ni cuotas de recuperación.

A pesar de tener mayor cobertura del seguro de salud, la etnia indígena tiene menor acceso a los servicios de salud y consulta preventiva en comparación con la población sin pertenencia étnica. Los indígenas refieren, en una mayor proporción que los demás grupos, que las razones para no acceder a los servicios de salud son la falta de dinero y el antecedente de que no le hubieran resuelto su problema en una atención previa (Tabla 4).

La pertenencia étnica, a excepción de la población negra, disminuye la probabilidad de tener buena percepción del estado de salud. Con respecto a la afiliación al seguro, la probabilidad aumenta para la población indígena y gitana, lo cual puede estar relacionado con los programas de priorización. La población indígena y la población negra tienen menor probabilidad de acceder a los servicios institucionales de salud con respecto a la población sin pertenencia étnica, en caso de tener un problema de salud. La utilización de servicios curativos y preventivos de salud es menor en las etnias que en la población general. En caso de necesitarlos, la población sin pertenencia étnica recibió en una mayor proporción todos los medicamentos con respecto a la población indígena, gitana y negra, como se observa en la tabla 4.

Tabla 4. Comparación de los indicadores de acceso a servicios de salud entre la población neara y la población sin pertenencia étnica en Bogotá. ECV-2003

\begin{tabular}{|c|c|c|c|c|c|c|}
\hline \multirow{2}{*}{$\begin{array}{c}\text { Indicador } \\
\text { (Proporción \%) }\end{array}$} & \multicolumn{5}{|c|}{ Poblacion } & \multirow[b]{2}{*}{$\begin{array}{c}\text { Velor de } \\
\text { P }\end{array}$} \\
\hline & Negra & Indigens & Gitana & Raizal & $\begin{array}{c}\text { Sin } \\
\text { pertenencia } \\
\text { cenica }\end{array}$ & \\
\hline $\begin{array}{l}\text { Población que reporta su } \\
\text { estado de salud como male }\end{array}$ & 15.3 & 34,1 & 40,6 & 35,1 & 21,0 & 0 \\
\hline $\begin{array}{l}\text { Población con necesidad } \\
\text { sentida en salud en el último } \\
\text { mes }\end{array}$ & 9,3 & 11,6 & 33,4 & 2,1 & 9,2 & $\infty, \infty 00$ \\
\hline $\begin{array}{l}\text { Población que refiere tener } \\
\text { diagnostico de enfermedad } \\
\text { crúnica }\end{array}$ & 10,1 & 26,3 & 41,0 & 10,3 & 14,0 & $<0,000$ \\
\hline $\begin{array}{l}\text { Población que refiere tener una } \\
\text { enfermedad grave }\end{array}$ & 5,6 & 12,2 & 5,4 & 7,6 & 10,7 & 0.001 \\
\hline $\begin{array}{l}\text { Población con necesidad } \\
\text { sentida en salud (SASH) }\end{array}$ & 22,3 & 21,5 & 10,6 & ND & 17,3 & $<0,000^{*}$ \\
\hline $\begin{array}{l}\text { Población que refiere tener } \\
\text { diagnostico de bajo peso } \\
\text { (SASH) }\end{array}$ & 3,1 & 3,5 & 3,5 & ND & 1,9 & $0,009^{\circ}$ \\
\hline $\begin{array}{l}\text { Población que refiere tener } \\
\text { diagndstico de diabetes } \\
\text { (SASH) }\end{array}$ & 1,8 & 3,2 & 1,5 & ND & 1,5 & $<0,000^{\circ}$ \\
\hline $\begin{array}{l}\text { Población que refiere tener } \\
\text { diagndstico do hipertension } \\
\text { arterial (SASH) }\end{array}$ & 5,8 & 5,9 & 5,6 & ND & 5,1 & $<0,000^{*}$ \\
\hline $\begin{array}{l}\text { Población que refiere tener } \\
\text { limitación ( } \mathrm{BASH} \text { ) }\end{array}$ & 2,3 & 3,2 & 1,8 & ND & 1,4 & $<0,000^{*}$ \\
\hline
\end{tabular}




\section{DISCUSIÓN}

Existen muy pocos estudios sobre equidad de etnia en Colombia y menos aún en Bogotá, y esto hace difícil contrastar los resultados de este estudio con esfuerzos previos. Se documentaron diferencias en el acceso a los servicios que no responden al reconocimiento de diferentes necesidades. Por el contrario, ellas expresan la ausencia de reconocimiento cultural. Como lo han mostrado otros estudios (18), la etnia es una categoría amplia, con diversos procesos organizativos que se agrupan con mayor precisión alrededor del concepto de "grupos étnicos". En vez de reconocer la especificidad étnica, parece confirmarse una discriminación excluyente en los servicios de salud, como se ha encontrado en otras investigaciones $(19,20)$. La cuantificación de la discriminación depende de las características de las preguntas, del contexto y la carga emocional, por lo que los hallazgos de esta investigación demandan profundización y un seguimiento mayor. La mayor vulnerabilidad de las etnias encontrada en este estudio concuerda con Bernal y colaboradores (21), quienes valoraron las inequidades en salud y las condiciones sociales de la población indígena y negra con respecto a la población general a nivel nacional, encontrando diferencias sistemáticas. Según las organizaciones étnicas entrevistadas, estas diferencias se explican por la violación de sus derechos colectivos, tales como la territorialidad, la autonomía y la identidad.

Las medicinas tradicionales son la primera opción terapéutica, aunque ese hallazgo no se ve reflejado en los resultados cuantitativos. Esto se podría explicar por el direccionamiento que hace la población para la pregunta de acceso a servicios institucionales, solamente a los problemas que no ha podido resolver. En el caso de la población negra, son conocidos varios estudios (22) que revelan mayor proporción de enfermedades cardiovasculares, diabetes, miomatosis uterina, anemia de células falciformes, entre otras. Algunas desigualdades derivadas de la pertenencia étnica y racial, como la anemia de células falciformes no serían necesariamente injustas, ya que depende fundamentalmente de factores biológicos. La mayor prevalencia de diabetes e hipertensión en población indígena y negra con respecto a población sin pertenencia étnica puede estar relacionada con los efectos combinados de la migración y la asimilación, la adopción de nuevos patrones de alimentación y la ausencia de factores protectores para el desenvolvimiento en el contexto urbano (23). Hay indicios, tanto en el ejercicio cuantitativo como en el cualitativo de esta investigación, de que el aseguramiento no es suficiente para garantizar la utilización de los servicios, ya que existen barreras a nivel cultural, administrativo, geográfico y financiero que limitan el acceso de las etnias, lo cual también se evidenció en el estudio de Gálvez y colaboradores (24) para la población indígena en Antioquia. 
$\mathrm{Al}$ igual que otros estudios (25), los hallazgos de este trabajo sugieren una interacción y potenciación entre diferentes procesos de exclusión, tales como las barreras socioeconómicas, la discriminación por razones étnicas, el racismo explícito e implícito, la falta de reconocimiento cultural, la migración forzada y el género. Estos procesos se articulan para profundizar la marginación estructural y la inequidad social que sufren los grupos étnicos en Bogotá *

\section{REFERENCIAS}

1. Organización Panamericana de la Salud. [Internet] Equidad en salud desde la perspectiva de la etnicidad. Disponible en: http://www.paho.org/spanish/ad/ge/ethnicity.htm. Consultado Febrero de 2007

2. London Health Observatory. [Internet] Health of Ethnic Minority Groups. Disponible en: http:// www.lho.org.uk/HEALTH_INEQUALITIES/EHIP.ASPX. Consultado Marzo de 2007.

3. Gros C. Políticas de la Etnicidad: identidad, estado y modernidad. Bogotá: ICANH, Instituto Colombiano de Antropologia e Historia; Diciembre de 2000.

4. Bustos F, Travassos C. Raca e saúde pública. Os dilemas da ciencia e da prática contemporánea. En: De Souza Minayo MC, Coimbra C (organizadores). Críticas e atuantes. Ciencias Sociais e Humanas em Saúde naAmérica Latina. Río de Janeiro: Editora Fiocruz; 2005. p. 461-471.

5. Reales L. Racismo y políticas públicas en Colombia. [Internet] Disponible en:http://www.eipcifedhop.org/EIPColombia/2005/afro_colombiens/Racismo.pdf Consultado Noviembre de 2007.

6. Flórez C, Medina C, Urrea F. Los costos de la exclusión social por raza o etnia en América Latina y el Caribe. Coyuntura Social. 2003; (29): 45-72.

7. Psacharopoulos G, Padrinos HG. Los pueblos indígenas y la pobreza en América Latina: un análisis empírico. Estudios sociodemográficos en pueblos indígenas, Serie E, No. 40 (LCl DEM/G.146), Santiago de Chile, División de Población, Centro Latinoamericano y Caribeño de Demografía (CELADE); 1994.

8. Krieger N. Does Racism Harm Health? Did Child Abuse Exist Before 1962?. On Explicit Questions, Critical Science, and Current Controversies: An Ecosocial Perspective. Am. J. Public Health. 2003; 93(2): 194-199.

9. McPherson KM, Harwood M, McNaughton HK. Ethnicity, equity, and quality: lessons from New Zealand. BMJ. 2003; 327(7412): 443-444.

10. Mendoza R. Utilización de los servicios de salud: una revisión sistemática sobre los factores relacionados. Cad Saúde Pública. 2001; 17(4): 819-832.

11. Hernández M. El pasado y el futuro del Observatorio para la Equidad en Calidad de Vida y Salud de Bogotá. En: Restrepo D. (Editor) Equidad y salud. Debates para la acción. Bogotá: Centro de Investigaciones para el Desarrollo-CID, Facultad de Ciencias Económicas, Universidad Nacional de Colombia; Secretaría Distrital de Salud; 2007. p. 173-194.

12. Fraser N. Lustitia Interrupta. Reflexiones críticas desde la posición «postsocialista». Universidad de los Andes. Facultad de Derecho. Santa Fe de Bogotá: Siglo del Hombre Editores; 1997.

13. Anand S. The Concern for Equity in Health. En: Anand S, Peter F, Sen AK. Public Health, Ethics, and Equity. Oxford: Oxford University Press; 2004. p. 15-20.

14. Andersen R, Aday LA. Access to medical care in the U.S.: realized and potential. Med Care. 1978 Jul;16(7):533-46.

15. Bronfman M, Castro R, Miranda C, Oviedo J, Zúñiga E. Del «cuánto» al «por qué»: la utilización de los servicios de salud desde la perspectiva de los usuarios. Salud Pública Mex 1997; 39: 442-450. 
16. Sen A. Health Achievement and Equity: External and Internal Perspectives. En Anand S, Peter F, Sen AK. Public Health, Ethics, and Equity. Oxford: Oxford University Press; 2004. p. 263268.

17. Jones R. Why do qualitative research? BMJ. 1995; 311(6996): 2.

18. Rodríguez, N, Jiménez, N. Estado del arte de la investigación sobre las comunidades de afrodescendientes y raizales en Bogotá D.C. Bogotá. Alcaldía Mayor de Bogotá. Instituto Distrital de Cultura y Turismo; Noviembre de 2006.

19. Universidad Nacional de Colombia. Centro de Estudios Sociales (CES). Mi gente en Bogotá. Estudio socioeconómico y cultural de los Afrodescendientes que residen en Bogotá. Bogotá. Alcaldía Mayor de Bogotá; Enero de 2002.

20. Research Network on Socioeconomic Status and Health Internet. [Internet] Disponible en http:// www.macses.ucsf.edu/Research/Psychosocial/notebook/discrimination.html\#Top Consultado en Marzo de 2008.

21. Bernal R, Cárdenas M. Race and Ethnic Inequality in Health and Health Care in Colombia. Working Paper Series No. 003413. Bogotá: FEDESARROLLO; 2005.

22. Monteiro S, Maio S. Etnicidae, Raca y Saùde No Brasil: Questoes e desafios. Em: De Souza Minayo MC, Coimbra C (organizadores). Críticas e atuantes. Ciencias Sociais e Humanas em Saúde na América Latina.. Río de Janeiro: Editora Fiocruz; 2005. p. 473-485.

23. Alvarado-Osuna C, Milian-Suazo F, Valles-Sánchez V. Prevalencia de diabetes mellitus e hiperlipidemias en indígenas otomíes. Salud Publica Mex 2001;43: 459-463.

24. GálvezA. El mañana que ya entró. La fecundidad en los pueblos indígenas de Antioquia. Medellín: Editorial Universidad de Antioquia; 2002.

25. Breihl J. Epidemiología Crítica. Ciencia Emancipadora e Interculturalidad. Buenos Aires: Lugar Editorial; 2003. 